\title{
Polymer:Fullerene Bimolecular Crystals for Near-Infrared Spectroscopic Photodetectors
}

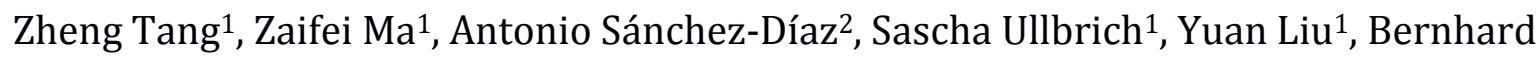
Siegmund ${ }^{1}$, Andreas Mischok ${ }^{1}$, Karl Leo ${ }^{1}$, Mariano Campoy-Quiles ${ }^{2}$, Weiwei Li ${ }^{3 *}$, Koen Vandewal ${ }^{1 *}$

${ }^{1}$ Dresden Integrated Center for Applied Physics and Photonic Materials (IAPP) and Institute for Applied Physics, Technische Universität Dresden, 01062, Dresden, Germany 2 Institut de Ciència de Materials de Barcelona (ICMAB-CSIC), Campus de la UAB, 08193, Bellaterra, Spain

${ }^{3}$ Beijing National Laboratory for Molecular Sciences, CAS Key Laboratory of Organic Solids, Institute of Chemistry, Chinese Academy of Sciences, Beijing 10090, P. R. China

Email: koen.vandewal@iapp.de; liweiwei@iccas.ac.cn

\begin{abstract}
Spectroscopic photodetection is a powerful tool in disciplines such as medical diagnosis, industrial process monitoring, or agriculture. However, its application in novel fields, including wearable and bio-integrated electronics is hampered by the use of bulky dispersive optics. Here, we employ solution-processed organic donor-acceptor blends in a resonant optical cavity device architecture for wavelength-tunable photodetection. While conventional photodetectors respond to above-gap excitation, the cavity device exploits weak subgap absorption of intermolecular charge-transfer states of the intercalating Poly[2,5-bis[3tetradecylthiophen-2-yl)thieno[3,2-b]thiophene] (PBTTT):[6,6]-Phenyl-C 61 -butyric acid methyl ester (PCBM) bimolecular crystal. This enables a highly wavelength selective, nearinfrared photoresponse with a spectral resolution down to $14 \mathrm{~nm}$, as well as dark currents and detectivities comparable with commercial inorganic photodetectors. A miniaturized spectrophotometer, comprising an array of narrowband photo-detectors is fabricated using blade-coated PBTTT:PCBM thin films with a thickness gradient. As application example, we demonstrate water transmittance spectral measured by this device.
\end{abstract}


Spectrophotometric methodologies are widely employed for resolving chemical compositions in medical diagnosis, environmental monitoring, agricultural production, astronomical investigation, imaging and surveillance. ${ }^{1,2}$ Traditional spectrometers are based on broadband photodetectors combined with diffraction gratings or dichroic prisms, ${ }^{3}$ characterized by a rather high structural complexity and cost. This can be circumvented using an array of photodetectors sensitive at specific wavelengths. Those, in turn, allow for miniaturization and new applications, such as wearable electronics and biomedical implementation. However, such arrays demand a significant advance in the development of photodetectors which should fulfill qualities such as narrow spectral response, continuously tunable detection wavelengths and an easy integration. Furthermore, the device architecture should be simple and achievable with a low-cost deposition techniques using abundant, nontoxic materials.

Solution processable organic bulk-heterojunctions $(\mathrm{BHJs})^{3}$ or metal-halide perovskites ${ }^{4}$ are in this respect interesting candidates, and several approaches to achieve wavelength selective photodetection using these materials have been recently proposed.5,6 For photo-active materials with tailored absorption spectra, high responsivities were achieved in the short-wavelength regime $(<700 \mathrm{~nm})$, with a spectral full-width at halfmaximum (FWHM) in the order of $100 \mathrm{~nm} \cdot{ }^{7-11}$ Narrower spectral responses were recently demonstrated through the use of thick active layers, resulting in carrier collection only for weakly absorbed wavelengths at the optical gap. ${ }^{12-14}$

Despite these recent developments, the reported narrowband detectors operate mostly in the visible range, with limited external quantum efficiencies (EQE) $(<10 \%)$. More importantly, the previously proposed device concepts are fundamentally limited on the widerange spectral tunability, which hinders its capability for spectroscopic applications.

An elegant alternative approach makes use of optical cavity effects. ${ }^{15}$ By sandwiching a 
weakly absorbing photo-active layer in-between two (partly) reflecting electrodes, narrow spectral resonances at specific wavelengths determined by the cavity thickness can be achieved. ${ }^{16-18}$ However, typical organic semiconductors employed for OPV present a high extinction coefficient that hinders the high quality, narrow, cavity resonances for above gap wavelengths (see below). In a donor-acceptor blend, the intermolecular charge-transfer (CT) states, on the other hand, provide weak absorption in the subgap region .Charge transfer state absorption with FWHM values down to $40 \mathrm{~nm}$ and EQE over $10 \%$ have recently been demonstrated in the 850-950 $\mathrm{nm}$ range, using cavity enhanced CT absorption of ZnPthalocyanine: $\mathrm{C}_{60}$ blends. An additional advantage of exploiting the intermolecular $\mathrm{CT}$ absorption band is that the detection wavelengths can extend far beyond those of the neat absorber materials. This allows for near infrared (NIR) detection without the synthesis of new organic absorbers. ${ }^{19}$

In this work, solution processed polymer cavity photodetectors that exploit theCT absorption, with low dark current and specific detectivities $\left(\boldsymbol{D}^{*}\right)$ comparable to commercial Si photodetectors are realized. By evaluating the cavity optics parameters, we propose materials selection rules that allow a simple device architecture in which a single absorber layer is sandwiched between mirroring electrodes. Using a PBTTT:PCBM blend, which, due to its intercalating nature has an appropriate CT absorption strength, we achieve a FWHM of $14 \mathrm{~nm}$ +- $10 \mathrm{~nm}$ ? and EQEs higher than $20 \%+-10 \%$ for wavelengths up to $960 \mathrm{~nm}$, which is more than $300 \mathrm{~nm}$ below the optical gap of PBTTT. Furthermore, prototype miniaturized spectrometers for the wavelength range $700 \mathrm{~nm}$ to $1100 \mathrm{~nm}$ are successfully constructed using a blade coated PBTTT:PCBM layer with a thickness wedge (i.e. a gradient). The simplicity in device construction and excellent performance along with the advantages of organic materials, such as low toxicity, flexibility and scalability, make this class of photodetectors highly interesting for innovative areas of spectroscopic applications, not 
compatible with present photodetecting technologies.

\section{Results}

\section{Resonant cavity photodetector device architecture}

Organic donor-acceptor BHJ blends are integrated into a resonant optical cavity device architecture. A schematic picture of the device architecture is shown in Figure 1a. The mirrors on both sides of the BHJ active layer simultaneously act as electrodes and are modified by ultrathin interlayers to achieve the desired work function. In this work, polyethylenimine $(\mathrm{PEIE})^{20}(<1 \mathrm{~nm})$ is used to lower the work function of the electron collecting bottom $\mathrm{Au}$. A 10 $\mathrm{nm} \mathrm{MoO}_{3}$ layer is used to provide hole selectivity to the top Ag electrode.

For such cavity devices, maximum field enhancement is reached at a resonance wavelength $\lambda_{m}$ which depends to a good approximation on the thickness $\boldsymbol{L}$ and refractive index $\boldsymbol{n}$ of the organic layer sandwiched between the metals ${ }^{17}$

$$
\lambda_{m}=\frac{2 L n}{m}
$$

Here the order of the resonance, $\boldsymbol{m}$, is a natural number $(>0)$. Simply adjusting the cavity thickness $\boldsymbol{L}$ allows a continuous tuning of the resonant wavelength of choice, later on employed for photodetection.

The FWHM of the photoresponse of such a cavity device is strongly related to the absorption strength of the active layer as well as parasitic absorption losses in the interlayers and mirrors. In an ideal cavity, the FWHM is proportional to the effective absorption coefficient $\boldsymbol{\alpha}$ of the cavity system, which includes the absorption coefficient of the active layer $\boldsymbol{a}_{\boldsymbol{a c t}}$, and parasitic absorptions (supplementary information, SI-2)

$$
\text { FWHM } \approx \frac{\alpha \lambda_{m}^{2}}{n \pi}
$$


Using equation (2), we can derive an upper limit for $\boldsymbol{a}_{\boldsymbol{a c t}}$, ensuring narrowband absorption with a FWHM of the EQE no larger than $50 \mathrm{~nm}$ (Figure 1b).

(a)

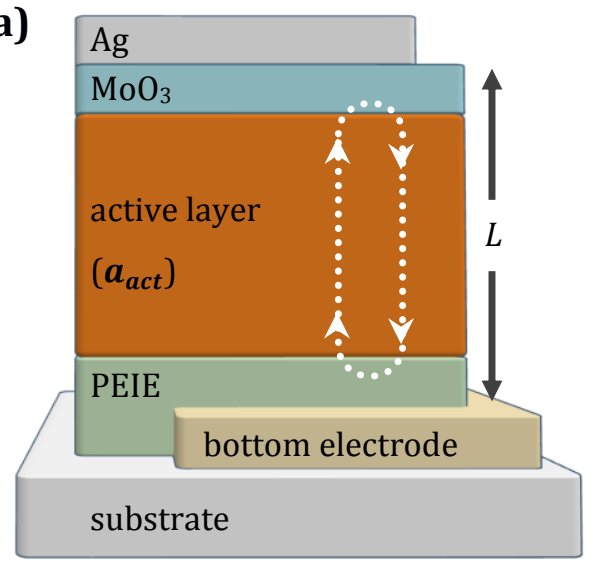

(c)

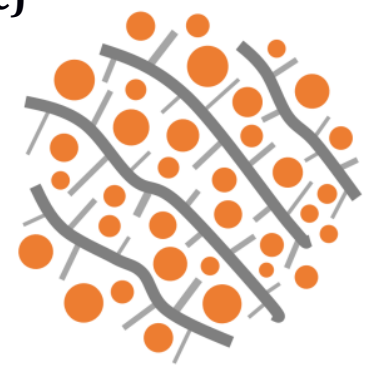

intercalating
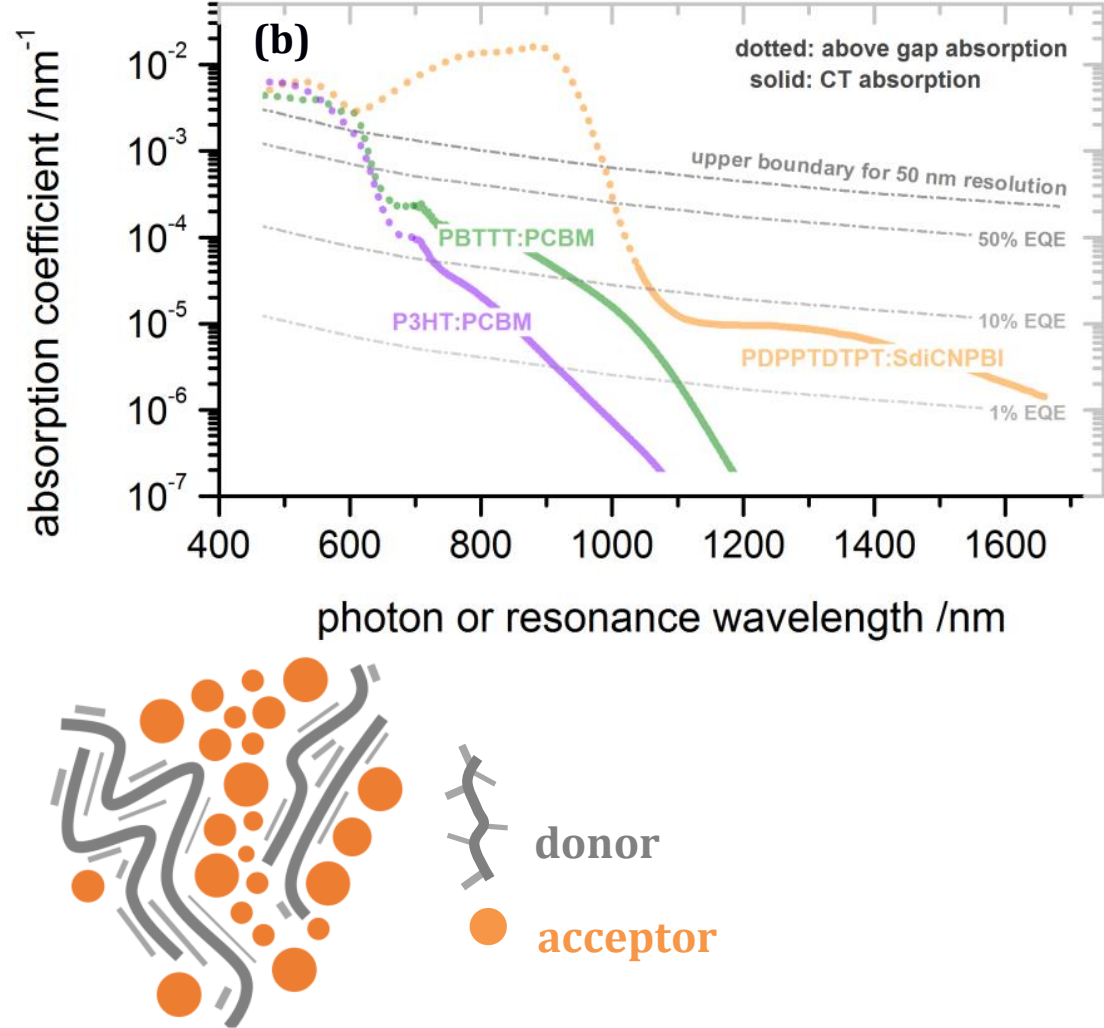

phase-separated

Figure 1. Device architecture and active materials properties. a: Schematic device architecture of a resonant cavity enhanced organic $\mathrm{BHJ}$ photodetector. The resonance wavelength is tuned by varying the thickness of the active layer. The metal-metal cavity detectors studied in this work have a semi-transparent Au bottom electrode and a non-transparent Ag top electrode. The devices based on DBRs have a semitransparent top Ag electrode, and a bottom DBR mirror modified by conductive PEDOT:PSS PH1000. b: Absorption coefficients of the blend active material systems of P3HT:PCBM 1:1 (weight ratio), PBTTT:PCBM 1:4, and PDPPTDTPT: SdiCNPBI 1:1. The upper and lower boundaries for the active layer absorption coefficient enabling a spectral FWHM $<50 \mathrm{~nm}$ and a maximum EQE above $1 \%, 10 \%$ and $50 \%$ are indicated. c: A schematic representation of an intercalated and non-intercalated BHJ donor (polymer)-acceptor system. The degree of intermixing between donor and acceptor material determines the CT absorption strength.

Using optical cavities, the EQE values at the resonance peaks can in principle be unity, but are practically reduced by parasitic absorption in the reflecting electrodes. The maximum achievable EQE (EQ $\left.\boldsymbol{E}_{\text {max }}\right)$ is given by,

$$
E Q E_{\text {max }}=\frac{a_{a c t}}{a_{a c t}+\frac{2 \tau}{L}}
$$

where $\boldsymbol{\tau}$ is the effective optical depth (unitless) responsible for absorption losses in the 
mirrors (Derived in SI-2). For noble metal mirrors, such as $\mathrm{Ag}$ and $\mathrm{Au}, \mathbf{2 \tau} \approx 0.05$. Equation (3) allows us to determine lower boundaries for $\boldsymbol{a}_{\text {act }}$. In Figure 1b, these are shown for a target $\boldsymbol{E} \boldsymbol{Q} \boldsymbol{E}_{\max }$ of $1 \%, 10 \%$, and $50 \%$.

\section{Materials selection and design rules}

A close look at Figure 1b shows that the absorption coefficient of a typical neat organic absorber $\left(\alpha>10^{-3} \mathrm{~nm}^{-1}\right)$ is too high to achieve narrowband $(<50 \mathrm{~nm})$ cavity enhancement. The subgap interfacial CT states formed at the interface between the donor and the acceptor, have intrinsically lower absorption coefficients, ${ }^{21-23}$ allowing, in principle, for narrower peaks. However, using noble metal electrodes, of which the parasitic absorption is much higher than typical CT absorption, considerably limits the peak EQE, especially for longer wavelengths. For instance, as shown in Figure 1b, the absorption coefficient of a standard BHJ blend based on poly(3-hexylthiophene-2,5-diyl) (P3HT) ${ }^{24}$ and PCBM falls in between the upper boundary for $50 \mathrm{~nm}$ FWHM and the lower one for $10 \%$ EQE, but only in a narrow wavelength range between 650 and $750 \mathrm{~nm}$.

To exploit the full potential of the device architecture shown in Figure 1a, the absorption strength of the CT state therefore needs to be increased. Since it is proportional to the density of CT complexes, or, the density of donor-acceptor contacts, ${ }^{25}$ blends with a high degree of intermixing between donor and acceptor materials are desired. In this respect, the semicrystalline polymer, PBTTT, ${ }^{26,27}$ has a structural advantage over P3HT, since it favors the intercalation of fullerene between its side chains and the formation of bimolecular crystals. ${ }^{28,29}$ While for P3HT:PCBM the lowest energy CT state is formed at the interface between aggregates of P3HT and PCBM, ${ }^{30}$ the highly intermixed PBTTT:PCBM blend has its lowest energy CT state within the co-crystal (Figure 1c). As compared to P3HT, PBTTT has a similar optical gap (absorption onset at $650 \mathrm{~nm}$ ) and a similar CT state energy $\left(\boldsymbol{E}_{\boldsymbol{C} \boldsymbol{T}} \approx 1.15 \mathrm{eV}\right.$, 
Figure S5, SI). However, PBTTT:PCBM shows significantly stronger CT absorption than P3HT:PCBM (Figure 1b), allowing for a wider spectral window (650 - $950 \mathrm{~nm}$ ) useful for resonant cavity effects, with expected EQEs over 10\%. To ensure efficient charge separation and transport in PBTTT:PCBM blends, a high load of acceptor material, in a 1:4 weight ratio, is used. $^{31}$

\section{Metal-Metal-cavity enhanced PBTTT:PCBM photodetectors}

We now demonstrate organic photodetectors based on BHJ systems with CT absorption enhanced by the simple metal-metal cavity (glass/Au $30 \mathrm{~nm} / \mathrm{PEIE} 1 \mathrm{~nm} /$ PBTTT:PCBM/MoO $10 \mathrm{~nm} / \mathrm{Ag} 100 \mathrm{~nm}$ ) of Figure $1 \mathrm{a}$. The thickness of the semitransparent $\mathrm{Au}$ mirror is optimized using a transfer matrix (TM) simulation, ${ }^{32}$ taking into account the effect of standing waves between the reflecting electrodes, and their parasitic absorption losses. As shown in Figure 2a, the measured EQE spectra of the PBTTT:PCBM detectors is in good agreement with the simulated results. The slightly lower experimental EQE peak values can be ascribed to non-unity IQE or thin film roughness, diminishing the cavity effect (Figure S6, SI). The continuous first order resonance wavelength tuning in the range between $700-1000$ $\mathrm{nm}$ is realized by varying the thickness of the active layer from 115 to $190 \mathrm{~nm}$. In this range, the EQEs are over $10 \%$, with FWHM $<50 \mathrm{~nm}$. Note that the above gap absorption of PBTTT contributes to the response at wavelengths below $700 \mathrm{~nm}$. To aid on the narrowband detection, the above gap absorption is easily suppressed by an additional PBTTT layer above the Au mirror, as shown in Figure S7 (SI).

Furthermore, metal-metal cavity photodetectors based on P3HT:PCBM system, as well as on PDPPTDTPT:SdiCNPBI ${ }^{33,34}$ are fabricated. For the latter, with a low $\boldsymbol{E}_{\boldsymbol{C}}(\approx 0.7 \mathrm{eV})$, detection wavelengths can be extended to a remarkable range of 1000 - $1700 \mathrm{~nm}$ (details in SI-3, SI-4). However, due to the reduced donor-acceptor intercalation, ${ }^{29}$ both blend systems 
result in photodetectors with low EQE $(<10 \%)$, in agreement with the results shown in Fig $1 b$.
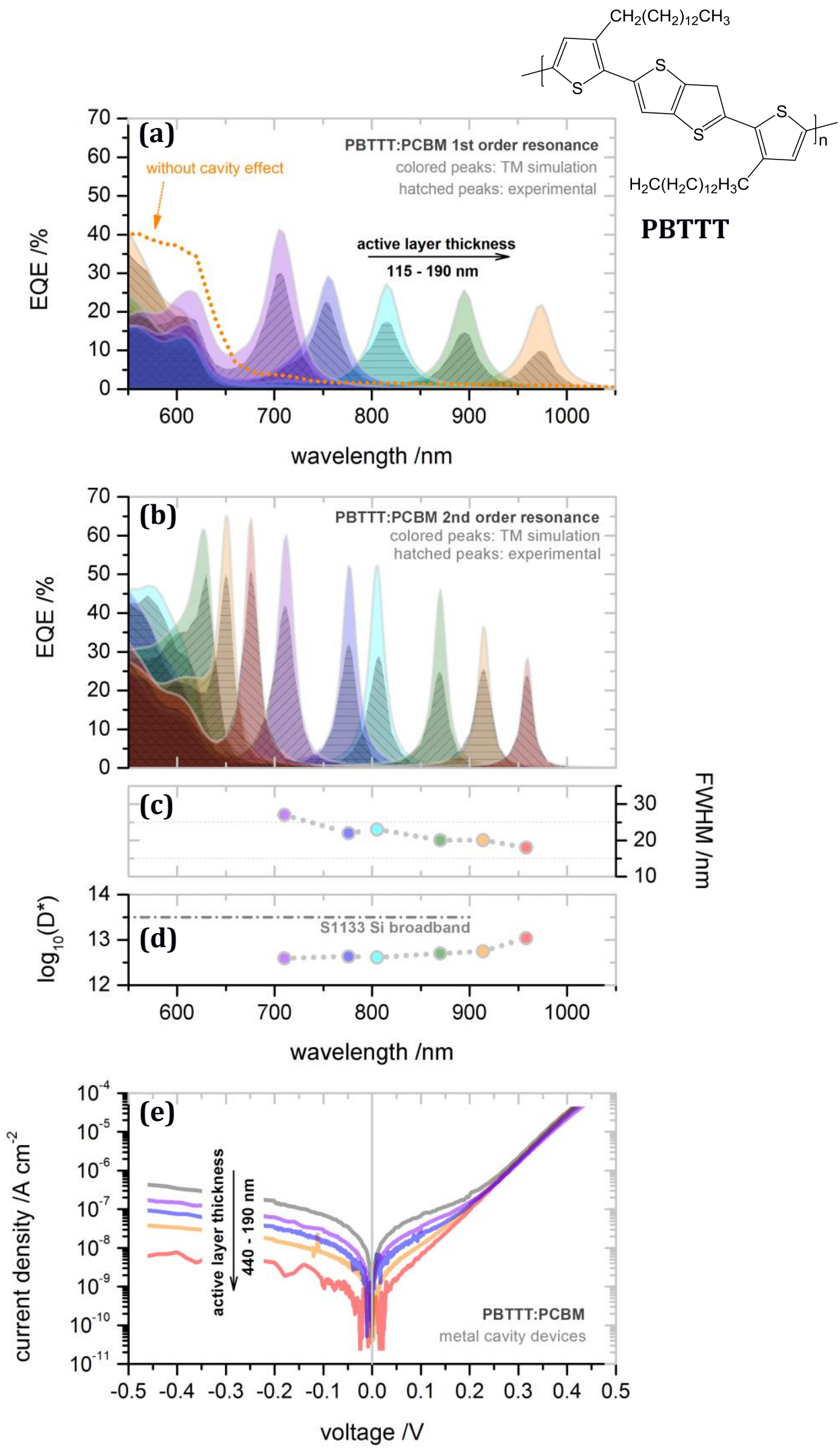
Figure 2. Performance of photodetectors based on PBTTT:PCBM (1:4) in a metal-metal cavity structure. Measured EQE and TM simulation predicted EQE (assuming an IQE of 100\%) for devices with an active layer thicknesses varied between a: $115-190 \mathrm{~nm}$ for first order resonances, and b: $290-440 \mathrm{~nm}$ for second order resonances. c: FWHM, and d: calculated specific detectivity $D^{*}$ (Jones) of the devices at second order resonances. The detectivity of a commercial Si based broadband photodetector (S1133, Hamamatsu) is also given for comparison. e: Dark $\boldsymbol{J} \boldsymbol{V}$ characteristic curves of the devices with different active layer thicknesses.

The TM simulations for the PBTTT:PCBM devices revealed that, at first order resonance, the EQE and spectral selectivity are still strongly limited by parasitic absorption in the electrodes (Figure S8, SI). According to equation 3, the effect of these parasitic absorption losses can be reduced by increasing the order of resonance $(\boldsymbol{m}>1)$, using thicker active layers. Figure 2 shows indeed a remarkable benefit of using second order resonances: we obtain much higher EQE values (20 - 40\%, Figure 2b) with FWHM values as small as $20 \mathrm{~nm}$ (Figure 2c). An added important advantage is the significant suppression in parasitic shunt current. ${ }^{35}$ As shown in Figure 2e, there is a clear dependence of the shunt resistance on the active layer thickness. From the current density-voltage (JV) characteristics of the device with a $400 \mathrm{~nm}$ active layer thickness, we derive a shunt resistance as high as $50 \mathrm{M} \Omega \mathrm{cm}^{2}$. In fact, shunt losses can barely be measured in the $\boldsymbol{J} \boldsymbol{V}$ curve of the thickest device, and the dark current under a small voltage bias is dominated by the dark saturation current related to the intrinsic diode properties of the device. This leads to very low Johnson and shot noise currents (Table S1, SI).

The specific detectivity $\boldsymbol{D}^{*}$ of a photodetector depends on the geometry of the electrodes, the shape of the active area, as well as the measurement circuit and shielding of the environmental noise sources. Focusing in this work on the device concept, we calculate the maximum achievable $\boldsymbol{D}^{*}$ at zero voltage bias (details in SI-1), assuming only the presence of Johnson noise. At the second order resonance wavelength close to $1000 \mathrm{~nm}, \boldsymbol{D}^{*}$ is over $10^{13}$ Jones (Figure 2d). This suggests that the performance of the cavity photodetectors based on PBTTT:PCBM approaches that of commercial broadband silicon detectors. ${ }^{35}$ 
Figure 3a shows the photocurrent of the PBTTT:PCBM device with its second order resonance of $1000 \mathrm{~nm}$, as a function of irradiance. When exciting at the resonance wavelength, the photoresponse is found to be strictly linear over 6.5 orders of magnitude, corresponding to a linear dynamic range (LDR) of at least $130 \mathrm{~dB}$, comparable to that of inorganic photodetectors. ${ }^{35,36}$ At the very low light intensities, the photoresponse is limited by the sensitivity of our measurement setup. For irradiances above $10 \mathrm{~mW} \mathrm{~cm}^{-2}$, a slightly sub-linear response due to bimolecular recombination ${ }^{37}$ is observed.

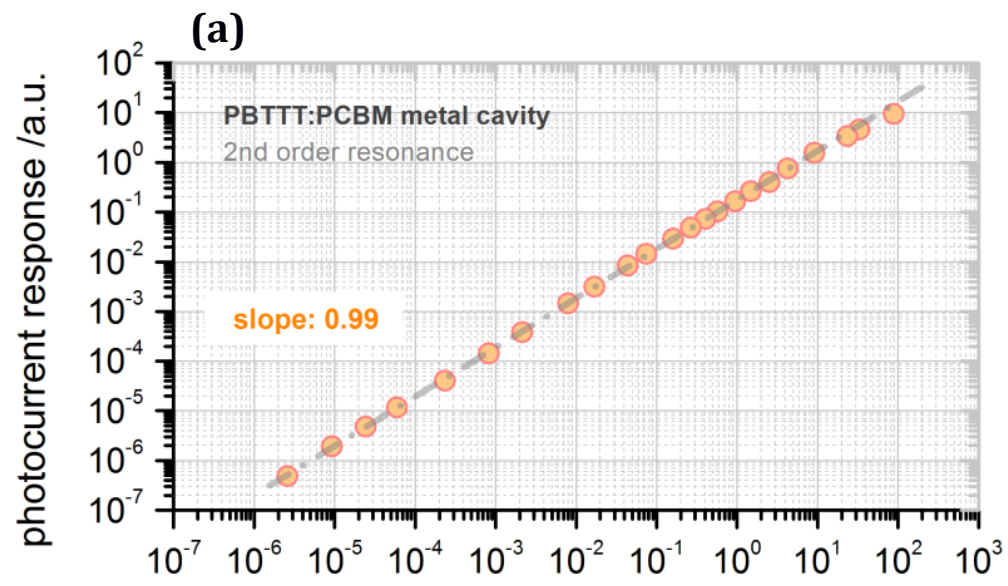

(b)

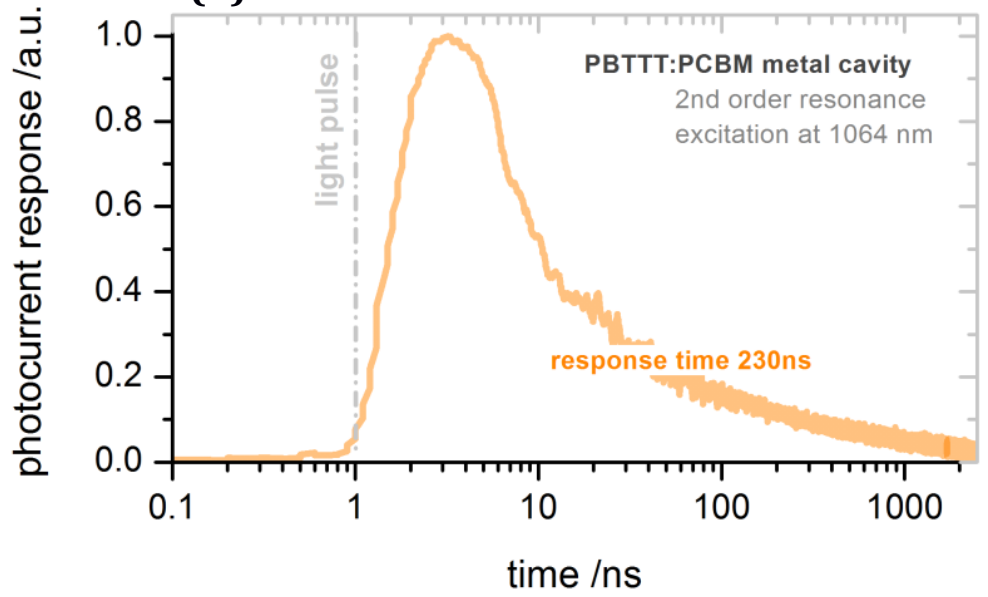

Figure 3. Photocurrent response of a photodetector based on PBTTT:PCBM (1:4) in a metalmetal cavity. The current response of a device with a resonance wavelength of $1000 \mathrm{~nm}$ (second order) is measured as a function of a: illumination intensity and $\mathbf{b}$ : time. The transient signal is recorded upon a NIR laser excitation starting at $1 \mathrm{~ns}$, with an excitation pulse length of $25 \mathrm{ps}$. The response time is derived from the $90 \%-10 \%$ signal fall time. The device has an active area of 0.5 $\mathrm{mm}^{2}$, a layer thickness of $440 \mathrm{~nm}$ and, was measured at short circuit conditions. 
The response time of the cavity device, with a second order resonance at $1000 \mathrm{~nm}$, (active layer thickness $=440 \mathrm{~nm}$, active area $=0.5 \mathrm{~mm}^{2}$ ) is measured under 25 ps pulsed laser excitation. As shown in Figure $3 \mathrm{~b}$, the device has a fall time (90\% to $10 \%)$ of $\approx 230 \mathrm{~ns}$, and a 3 $\mathrm{dB}$ bandwidth at short circuit condition of over $1 \mathrm{MHz}$, which is certainly sufficiently fast for a detector array readout.

\section{Photodetectors based on Distributed-Bragg-Reflectors}

The use of noble metal thin films acting simultaneously as (semi-transparent) mirror and electrode introduces a considerable amount of parasitic absorption, limiting device performance, even for the second order resonance devices. Further improvement thus requires alternative (non-metal) reflecting electrodes with higher reflectivity and lower parasitic absorption losses. Below, we demonstrate devices in which high conductivity PEDOT:PSS PH1000 coated Distributed-Bragg-Reflectors (DBR) are used as the back reflecting electrode. We constructed two DBRs consisting of 21 alternating $\mathrm{TiO}_{2}$ and $\mathrm{SiO}_{2}$ layers $\left(\mathrm{TiO}_{2}\right.$ being the top layer). ${ }^{38}$ Due to the high contrast in refractive index between the $\mathrm{TiO}_{2}$ and the $\mathrm{SiO}_{2}$ layers, a reflectance over $99.9 \%$ within a stopband is possible. By optimizing the dielectric layer thicknesses, two sets of DBRs with stopbands in the range of $650-800 \mathrm{~nm}$ and $750-900 \mathrm{~nm}$ are fabricated (Figure S9, SI), well suited for the CT state absorption of PBTTT:PCBM.

The DBR cavity devices have an architecture of glass/DBR/PEDOT:PSS PH1000 (20 $\mathrm{nm}) / \mathrm{PEIE}(1 \mathrm{~nm}) / \mathrm{PBTTT}_{\mathrm{PCBM}} / \mathrm{MoO}_{3}(10 \mathrm{~nm}) / \mathrm{Ag}(30 \mathrm{~nm})$. The PEIE modified, electron collecting PEDOT electrode ${ }^{39}$ is thin enough to avoid significant parasitic absorption losses, while still being sufficiently conductive in the lateral direction. EQEs of second order resonance devices on DBRs are shown in Figure 4a. Compared to photodetectors based on a metal-metal cavity, the spectral selectivity of the DBR devices is further improved. The 
spectral line FWHM value is further reduced to $14 \mathrm{~nm}$ (Figure $4 \mathrm{~b}$ ), which is the lowest value for organic narrowband photodetectors to date.

It should be further stressed that the high spectral selectivity is achieved without compromising on specific detectivity. In fact, we find that the $\boldsymbol{J V}$ curves of the detectors based on DBRs are similar to those based on metal mirrors with the same active materials system (Figure S10, SI). This leads, also for the DBR based detectors, to a calculated specific detectivity close to $10^{13}$ Jones (Figure 4c).

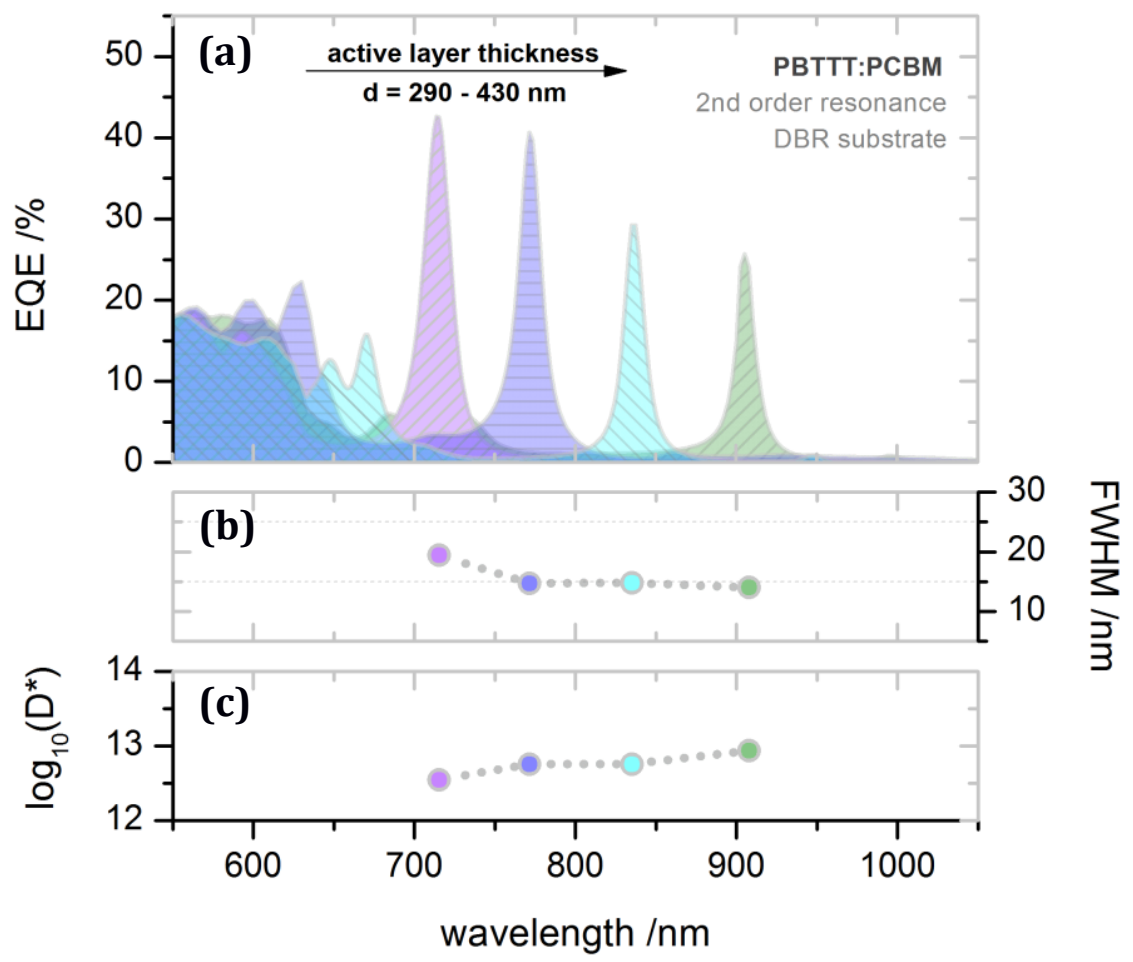

Figure 4. Performance of cavity enhanced photodetectors based on DBRs and PBTTT:PCBM (1:4). a: EQE, b: FWHM and c: specific detectivity $D^{*}$ (Jones) for devices with a second order resonance. Device architecture: glass/Ag $(30 \mathrm{~nm}) / \mathrm{MoO}_{3}(10 \mathrm{~nm}) /$ active layer/PEIE $(1 \mathrm{~nm}) /$ PEDOT:PSS PH1000 $(20 \mathrm{~nm}) / \mathrm{DBR}$.

\section{Miniaturized spectrometers based on PBTTT:PCBM CT absorption}

Figure 5a displays a schematic picture and a photograph of a proof-of-concept miniature spectrometer consisting of an array of metal-metal cavity photodetectors based on PBTTT:PCBM. Due to the thickness gradient in the active layer, each pixel has a different resonance wavelength, enabling spectroscopic photodetection with a resolution related to the FWHM of the individual photodetectors. The thickness gradient of the PBTTT:PCBM active 
layer is achieved by blade coating with a linearly decreasing coating speed. Coating conditions (details in SI-1) are optimized to achieve a thickness variation from $100 \mathrm{~nm}$ to $200 \mathrm{~nm}$ within a distance of $6 \mathrm{~cm}$, resulting in first order resonance wavelengths in the range of 700-1100 nm. The spectral responses of the photodetectors are given in Figure S11 (SI).
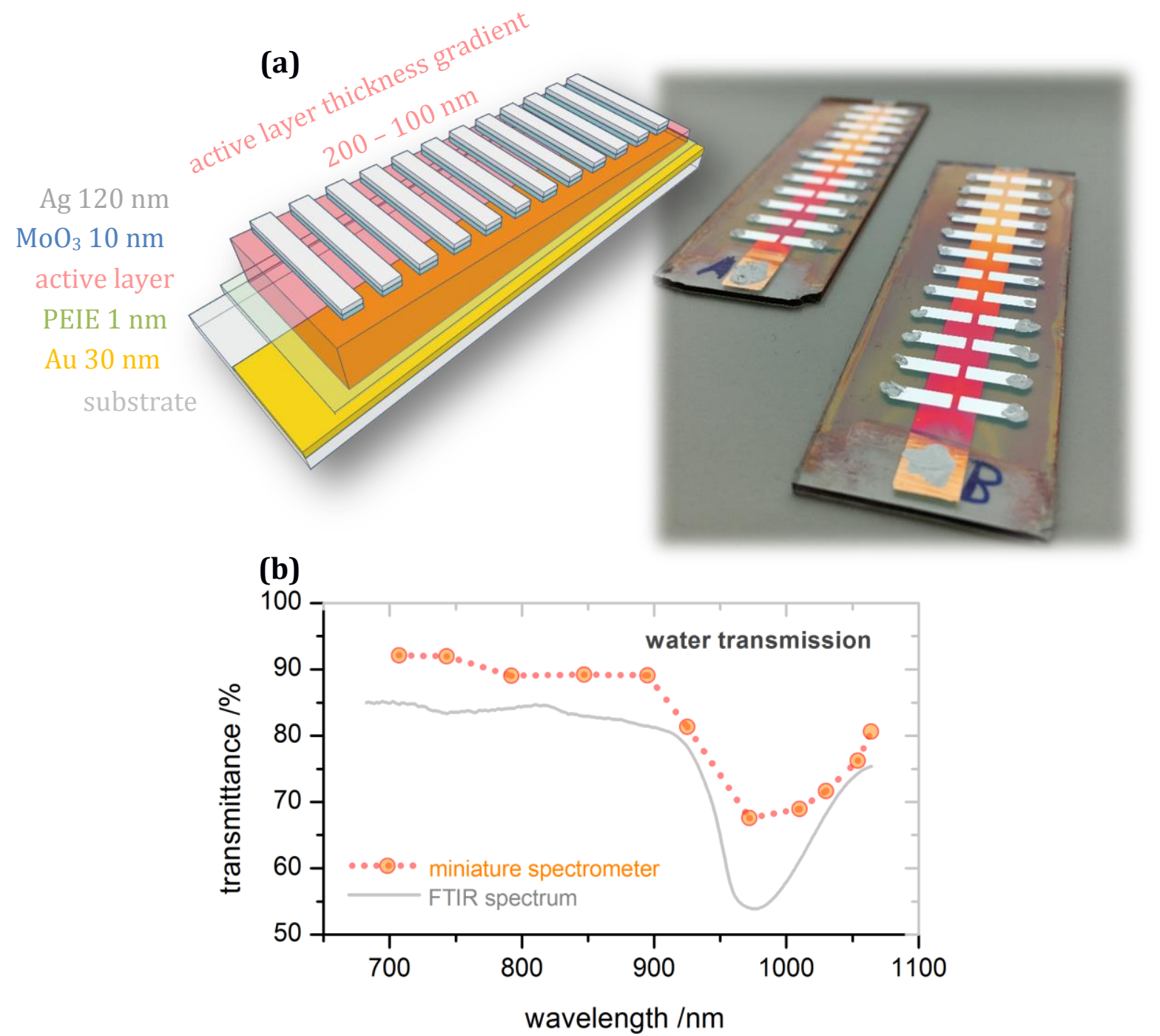

Figure 5. Miniature NIR spectrometer with a thickness wedged PBTTT:PCBM layer in a metal-metal cavity. a: A photograph of the spectrometers fabricated in this work; and a schematic picture of the device architecture. The spectrometer is based on a single wedged active layer, resulting in different resonance wavelengths for each individual detecting pixel. The change in active layer color is the result of the gradually changing interference condition. $\mathbf{b}$ : Water transmittance spectrum measured by the spectrometer constructed in this work in comparison to a spectrum measured by a commercial Fourier transform infrared spectrometer.

In order to demonstrate that this device has a sufficient spectral resolution for a potential application in water detection, Figure $5 \mathrm{~b}$ shows a measurement of the transmittance spectrum of water obtained using the miniature spectrometer and a xenon lamp as the light 
source. The transmission feature at $970 \mathrm{~nm}$, corresponding to the first vibrational overtone of the $\mathrm{O}-\mathrm{H}$ bond absorption, can be clearly identified and is in agreement with the results found with a commercial system. Upon further miniaturization, such organic cavity spectrometers can thus provide a simple and cheap alternative for inline water monitoring, a key component in wood, paper, concrete or pharmaceutical industry.

\section{Discussion}

The presented proof-of-concept device demonstrates a combination of a wide spectroscopic photodetection and good device compactness with potential for a fast readout, as well as simple and cheap fabrication method, which cannot be easily achieved with other technologies. A further miniaturization of the spectrometer will require reduced photodetecting pixel size and spacing. Alternative deposition techniques such as slot-die or inkjet printing, ${ }^{40}$ are potential candidates to increase the active layer thickness gradient slope and specially increase the mass production of such devices.

The performance of the miniature spectrometer is limited by parasitic absorption losses in the metal electrodes. We have demonstrated that with second order resonances (Figure 2), or DBRs (Figure 4), the performance of organic cavity detectors based on PBTTT:PCBM CT absorption is improved, as compared to previously reported narrowband photodetectors based on organic or perovskite materials. ${ }^{13,14}$ Furthermore, as compared to previously reported cavity detectors based on vacuum deposited small molecules, ${ }^{18,19}$ we have shown significantly enhanced device spectral selectivity, lower dark currents, easier spectral tunability and a simple device architecture using polymer-fullerene blends. In fact, the solution processed $\mathrm{BHJ}$ cavity detectors have in many aspects a comparable performance as commercial silicon based detectors, ${ }^{35}$ with the latter lacking spectral tunability and selectivity. 
The presented approach is particularly interesting in the NIR region of the electromagnetic spectrum, where the CT bands of most of donor-acceptor combinations developed for organic solar cells are found. ${ }^{21,41}$ By varying the combination of donor and acceptor materials, the CT absorption can be tuned up to $1700 \mathrm{~nm}$, as we demonstrated with the PDPPTDTPT:SdiCNPBI system (details in SI-4), to the best of our knowledge this is the broadest tunability rangefound in organic narrowband photodetectors. ${ }^{42-46}$ More importantly, our novel approach for extending the detection range of a photodetector is considerably simpler than the conventional methods that require the developments of new absorber materials with varying band gaps, for which a suitable acceptor needs to be found.

We expect future research to focus on performance improvements for wavelengths beyond $1100 \mathrm{~nm}$. New, promising $\mathrm{BHJ}$ candidates being used as the active material system for cavity enhanced detectors should not only have proper energy levels, but also, intercalate significantly on the molecular level to increase the number of interfacial CT states. While the PBTTT:PCBM co-crystal has been successfully used as model system for fundamental studies on organic photovoltaics, we anticipate future applications of such intercalating crystals in cavity detectors and spectrometers, spanning the entire visible to NIR region.

\section{Methods}

Detailed in supplementary information.

\section{Acknowledgement}

We thank Prof. Olle Inganäs for discussions and use of his lab for carrying out parts of this work. This work was supported by the Alexander von Humboldt-Foundation, through fellowships to Z.T. and Z.M., and the German Federal Ministry for Education and Research (BMBF) through the InnoProfile project “Organische p-i-n Bauelemente 2.2”. W. Li acknowledges financial support by the National Natural Science Foundation of China 
(51603209, 21574138) and the Strategic Priority Research Program (XDB12030200) of the Chinese Academy of Sciences. A.M. acknowledges financial support by the Deutsche Forschungsgemeinschaft, project number LE747/53-1. A.S.D. and M.C.Q. thank the European Research Council for financial support through grant CoG648901 and Ministerio de Economía y Competitividad of Spain through the "Severo Ochoa" Programme for Centers of Excellence in R\&D (SEV-2015-0496) and project MAT2015-70850-P.

\section{Author contributions}

The project was designed by Z.T. and K.V., and supervised by K.V.

Z.T. and K.V. derived the analytic equations. Z.T. and Z.M. fabricated and optimized the metalmetal cavity detectors. DBR mirrors constructed and optimized by Y.L., A.M., supervised by K.L., and Z.T. constructed the DBR based detectors. The transfer matrix simulations were done by Z.T. using the optical constants measured by Z.M. and A.M. EQE and JV measurements were done by Z.T. Response time measurements and linear dynamic range measurement were done by Z.T., S.U., and B.S. AFM images were taken by Z.M. PDPPTDTPT and SdiCNPBI were provided by W.L. Fabrication and characterization of miniaturized spectrometers were done by Z.T., and A.S.D., supervised by M.C.Q.

Z.T. wrote the manuscript with K.V., and all authors contributed to the discussion and the finalizing of the manuscript.

\section{References}

1. Bacon, C. P., Mattley, Y. \& DeFrece R. Miniature spectroscopic instrumentation: Applications to biology and chemistry. Rev. Sci. Instrum. 75, 1-16 (2003).

2. Arquer, F. P. G. de, Armin, A., Meredith, P. \& Sargent, E. H. Solution-processed semiconductors for next-generation photodetectors. Nat. Rev. Mater. 2, 16100 (2017). 
3. Yu, G., Gao, J., Hummelen, J. C., Wudl, F. \& Heeger, A. J. Polymer Photovoltaic Cells: Enhanced Efficiencies via a Network of Internal Donor-Acceptor Heterojunctions. Science 270, 17891791 (1995).

4. Kojima, A., Teshima, K., Shirai, Y. \& Miyasaka, T. Organometal Halide Perovskites as VisibleLight Sensitizers for Photovoltaic Cells. J. Am. Chem. Soc. 131, 6050-6051 (2009).

5. Baeg, K. J., Binda, M., Natali, D., Caironi, M. \& Noh, Y.-Y. Organic Light Detectors: Photodiodes and Phototransistors. Adv. Mater. 25, 4267-4295 (2013).

6. Jansen-van Vuuren, R. D., Armin, A., Pandey, A. K., Burn, P. L. \& Meredith, P. Organic Photodiodes: The Future of Full Color Detection and Image Sensing. Adv. Mater. 28, 47664802 (2016).

7. Rauch, T. et al. Near-infrared imaging with quantum-dot-sensitized organic photodiodes. Nat. Photonics 3, 332-336 (2009).

8. Jansen van Vuuren, R. et al. Determining the absorption tolerance of single chromophore photodiodes for machine vision. Appl. Phys. Lett. 96, 253303 (2010).

9. Higashi, Y., Kim, K. S., Jeon, H. G. \& Ichikawa, M. Enhancing spectral contrast in organic redlight photodetectors based on a light-absorbing and exciton-blocking layered system. J. Appl. Phys. 108, 034502 (2010).

10. Lee, K. H. et al. Dynamic Characterization of Green-Sensitive Organic Photodetectors Using Nonfullerene Small Molecules: Frequency Response Based on the Molecular Structure. J. Phys. Chem. C 118, 13424-13431 (2014).

11. Han, M. G. et al. Narrow-Band Organic Photodiodes for High-Resolution Imaging. ACS Appl. Mater. Interfaces 8, 26143-26151 (2016). 
12. Armin, A., Vuuren, R. D. J., Kopidakis, N., Burn, P. L. \& Meredith, P. Narrowband light detection via internal quantum efficiency manipulation of organic photodiodes. Nat. Commun. 6, 6343 (2015).

13. Lin, Q., Armin, A., Burn, P. L. \& Meredith, P. Filterless narrowband visible photodetectors. Nat. Photonics 9, 687-694 (2015).

14. Fang, Y., Dong, Q., Shao, Y., Yuan, Y. \& Huang, J. Highly narrowband perovskite singlecrystal photodetectors enabled by surface-charge recombination. Nat. Photonics 9, 679$686(2015)$.

15. Vahala, K. J. Optical microcavities. Nature 424, 839-846 (2003).

16. Kishino, K. et al. Resonant cavity-enhanced (RCE) photodetectors. IEEE J. Quantum Electron. 27, 2025-2034 (1991).

17. Ünlü, M. S. \& Strite, S. Resonant cavity enhanced photonic devices. J. Appl. Phys. 78, 607-639 (1995).

18. An, K. H., O'Connor, B., Pipe, K. P. \& Shtein, M. Organic photodetector with spectral response tunable across the visible spectrum by means of internal optical microcavity. Org. Electron. 10, 1152-1157 (2009).

19. Siegmund, B. et al. Organic narrowband near-Infrared photo-detectors based on intermolecular charge-transfer absorption. Nat. Commun., accepted.

20. Zhou, Y. et al. A Universal Method to Produce Low-Work Function Electrodes for Organic Electronics. Science 336, 327-332 (2012).

21. Vandewal, K., Tvingstedt, K., Gadisa, A., Inganäs, O. \& Manca, J. V. On the origin of the open-circuit voltage of polymer-fullerene solar cells. Nat. Mater. 8, 904-909 (2009). 
22. Vandewal, K. Interfacial Charge Transfer States in Condensed Phase Systems. Annu. Rev. Phys. Chem. 67, 113-133 (2016).

23. Vandewal, K. et al. Efficient charge generation by relaxed charge-transfer states at organic interfaces. Nat. Mater. 13, 63-68 (2014).

24. Padinger, F., Rittberger, R. s. \& Sariciftci, N. s. Effects of Postproduction Treatment on Plastic Solar Cells. Adv. Funct. Mater. 13, 85-88 (2003).

25. Vandewal, K., Tvingstedt, K., Gadisa, A., Inganäs, O. \& Manca, J. V. Relating the opencircuit voltage to interface molecular properties of donor:acceptor bulk heterojunction solar cells. Phys. Rev. B 81, 125204 (2010).

26. McCulloch, I. et al. Liquid-crystalline semiconducting polymers with high chargecarrier mobility. Nat. Mater. 5, 328-333 (2006).

27. Buchaca-Domingo, E. et al. Direct Correlation of Charge Transfer Absorption with Molecular Donor:Acceptor Interfacial Area via Photothermal Deflection Spectroscopy. J. Am. Chem. Soc. 137, 5256-5259 (2015).

28. Mayer, A. C. et al. Bimolecular Crystals of Fullerenes in Conjugated Polymers and the Implications of Molecular Mixing for Solar Cells. Adv. Funct. Mater. 19, 1173-1179 (2009).

29. Miller, N. C. et al. Factors Governing Intercalation of Fullerenes and Other Small Molecules Between the Side Chains of Semiconducting Polymers Used in Solar Cells. Adv. Energy Mater. 2, 1208-1217 (2012).

30. Vandewal, K. et al. Varying polymer crystallinity in nanofiber poly(3-alkylthiophene): PCBM solar cells: Influence on charge-transfer state energy and open-circuit voltage. Appl. Phys. Lett. 95, 123303 (2009). 
31. Parmer, J. E. et al. Organic bulk heterojunction solar cells using poly(2,5-bis(3tetradecyllthiophen-2-yl)thieno[3,2,-b]thiophene). Appl. Phys. Lett. 92, 113309 (2008).

32. Pettersson, L. A. A., Roman, L. S. \& Inganäs, O. Modeling photocurrent action spectra of photovoltaic devices based on organic thin films. J. Appl. Phys. 86, 487-496 (1999).

33. Hendriks, K. H., Li, W., Wienk, M. M. \& Janssen, R. A. J. Small-Bandgap Semiconducting Polymers with High Near-Infrared Photoresponse. J. Am. Chem. Soc. 136, 12130-12136 (2014).

34. Yu, Y. et al. A perylene bisimide derivative with a LUMO level of $-4.56 \mathrm{eV}$ for nonfullerene solar cells. J. Mater. Chem. C 4, 4134-4137 (2016).

35. Armin, A. et al. Thick junction broadband organic photodiodes. Laser Photonics Rev. 8, 924-932 (2014).

36. Guo, F., Xiao, Z. \& Huang, J. Fullerene Photodetectors with a Linear Dynamic Range of 90 dB Enabled by a Cross-Linkable Buffer Layer. Adv. Opt. Mater. 1, 289-294 (2013).

37. Lakhwani, G., Rao, A. \& Friend, R. H. Bimolecular Recombination in Organic Photovoltaics. Annu. Rev. Phys. Chem. 65, 557-581 (2014).

38. Dynamics of a high-Q vertical-cavity organic laser. Appl. Phys. Lett. 87, 181108 (2005).

39. Tang, Z. et al. Fully-solution-processed organic solar cells with a highly efficient paperbased light trapping element. J. Mater. Chem. A 3, 24289-24296 (2015).

40. Krebs, F. C. Fabrication and processing of polymer solar cells: A review of printing and coating techniques. Sol. Energy Mater. Sol. Cells 93, 394-412 (2009).

41. Infrared photocurrent response of charge-transfer exciton in polymer bulk heterojunction. Appl. Phys. Lett. 92, 083504 (2008). 
42. Gong, X. et al. High-Detectivity Polymer Photodetectors with Spectral Response from $300 \mathrm{~nm}$ to $1450 \mathrm{~nm}$. Science $\mathbf{3 2 5}, 1665-1667$ (2009).

43. Arnold, M. S. et al. Broad Spectral Response Using Carbon Nanotube/Organic Semiconductor/C60 Photodetectors. Nano Lett. 9, 3354-3358 (2009).

44. Zimmerman, J. D. et al. Use of additives in porphyrin-tape/C60 near-infrared photodetectors. Org. Electron. 12, 869-873 (2011).

45. Young, M. et al. Organic Heptamethine Salts for Photovoltaics and Detectors with NearInfrared Photoresponse up to 1600 nm. Adv. Opt. Mater. 4, 1028-1033 (2016).

46. London, A. et al. Donor-Acceptor Polymers with Tunable Infrared Photoresponse. Polym. Chem. (2017). doi:10.1039/C7PY00241F 